\title{
Surgical Hip Dislocation for the Treatment of Slipped Upper Femoral Epiphysis - Demonstration of Surgical Technique
}

 \\ Abu Rumman, $M D^{2}$ \\ ${ }^{1}$ Assistant Professor and Consultant Orthopaedic Surgeon, Department of Trauma and Orthopaedics, \\ Faculty of Medicine, The Hashemite University, Zarqa, Jordan \\ ${ }^{2}$ Orthopaedic Resident, Department of Trauma and Orthopaedics, Prince Hamza Hospital, Amman, Jordan
}

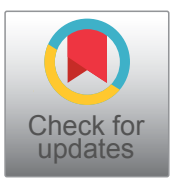

Surgical hip dislocation for treatment of hip intra-articular pathology has been popularized by the Bernese group over the last few years. This approach provides excellent and safe exposure of the hip joint without compromising the femoral head blood supply. It is, however a very demanding approach and requires surgical experience and attention to detail.

Surgical hip dislocation for treatment of severe cases of Slipped Upper Femoral Epiphysis (SUFE) is even more technical and has few more steps. Severe slips, by definition, can compromise the blood supply and therefore special care must be taken to maintain the remaining vascularity of the femoral head. In this article we will demonstrate in detail the same approach described by Ganz, et al. going through all the surgical steps and demonstrating those using intra-operative photos. The aim of this article is to make the approach clearer to orthopaedic surgeons dealing with this complex problem.

\section{Introduction}

Slipped Upper Femoral Epiphysis (SUFE) affects about 1 in 100,000 children [1]. It is usually a disease of the adolescence but can affect younger children occasionally. The condition varies in severity and in chronicity. In situ pinning has been traditionally labelled as the gold standard for treatment of all cases regardless of the severity of the slip [2]. In situ pinning seems to give good results for mild and may be moderate slips. However, bad results have been reported for severe slips and subsequent surgeries did not seem to resolve patients' complains [3]. Many of these patients continue to suffer and require arthroplasty at a very young age. Furthermore, in situ pinning is not necessarily an easy operation in severe slips and some complications have been reported such as chondrolysis, fractures, avascular necrosis etc. in addition to the arguable remodeling potential of the proximal femur after slippage of the femoral head $[2,4]$.

Other treatment options included reductions; closed or open and osteotomies. Closed reductions are not advised in most cases due to the very significant of disrupting the remaining blood supply which will inevitably lead to avascular necrosis. Basal neck and inter-trochanteric osteotomies are technically easier to do but are farther away from the CORA and lead to non-anatomical correction and a further deformity especially in severe slips [5]. Intra-articular osteotomies to achieve a reasonable reduction are more demanding and carry higher risk of avscaular necrosis but in principle correct the original deformity and can achieve better results [4]. The slip site can be approached via an anterior approach e.g. Fish Osteotomy but full access is not possible especially to the posterior aspect of the neck where the slipped femoral head is lying, and anatomical reduction is difficult and carries a very high incidence of AVN. The original Dunn osteotomy utilizes a posterior KockerLangenbeck approach which is slightly better but the created retinacular flaps are short which limits the access and the reduction and again risks the development of AVN.

The concept of 'Safe Surgical Hip Dislocation' was introduced by Ganz, et al. in order to directly visualize and deal with intra-articular hip pathology without compromising the vascularity of the femoral head $[6,7]$. The Bernese group initially published their technique of dislocating the hip joint after performing an anterior Z-shaped capsulotomy to protect the blood supply. Subsequently the same group described the same approach to perform the 'modified Dunn osteotomy' to treat severe slips $[4,8]$. We noticed that most surgeons are very hesitant to perform this approach because of its technicalities. In this article we will explain and demonstrate with intra-operative photos all steps of the approach hoping to clarify it for orthopaedic surgeons who deal with such cases.

\footnotetext{
*Corresponding author: Saeed Al-Naser, MD, Assistant Professor and Consultant Paediatric Orthopaedic Surgeon, Department of Trauma and Orthopaedics, Faculty of Medicine, The Hashemite University, Zarqa, Jordan, Tel: 00962791130920, Fax: 0096265682237
}

Accepted: November 10, 2020

Published online: November 12, 2020

Citation: Al-Naser S, Altamimi AAR, Gharaibeh MA, et al. (2020) Surgical Hip Dislocation for the Treatment of Slipped Upper Femoral Epiphysis - Demonstration of Surgical Technique. J Orthop Surg Tech 3(2):205-210 




Figure 1: A) AP Xray of the hips taken with the patient's trousers on. The buckle of the belt is covering the Right hip therefore missing the diagnosis; B) Frog lateral X-ray of both hips showing severe SUFE of the Right Hip; C) Lateral hip X-ray showing severe SUFE of the Right Hip; D) MRI Axial Cut showing the severity of the slip. The femoral head is severely displaced and is sitting at nearly right angle to the femoral head. The red arrow shows the direction of the screw if in situ pinning is attempted. The screw will need to go through the posterior femoral neck to get into the centre of the femoral head. This may damage any remaining blood supply to the femoral head; E) MRI Axial Cut showing some callus at the head-neck junction posteriorly (red arrow) indicating this is a chronic slip. In this patient there was pain over two months which became severe over the previous 24-hours therefore was classified as 'Acute on chronic' slip.

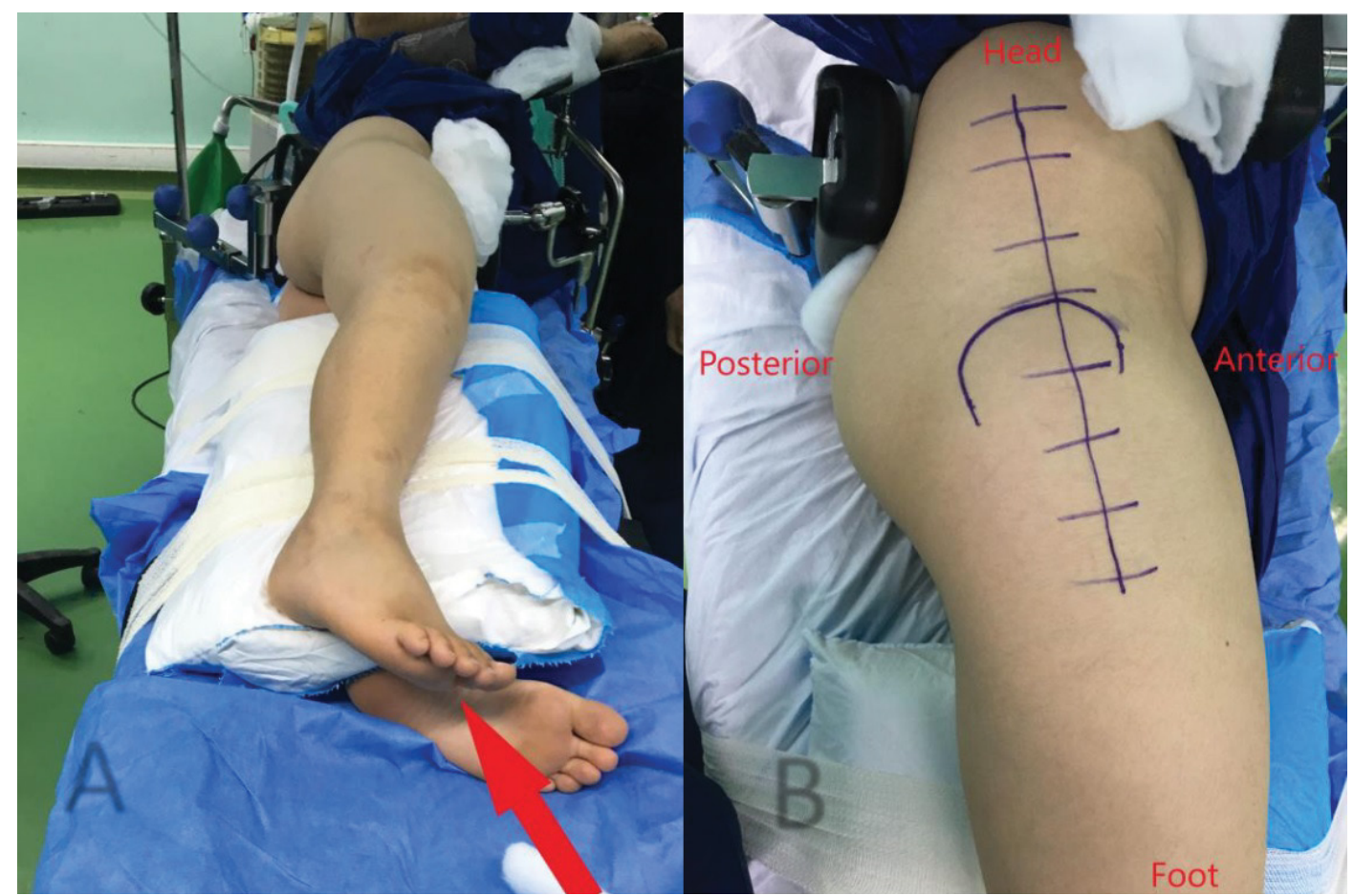

Figure 2: Positioning and incision. A) Positioning of the patient. The patient is positioned in the lateral decubitus position with the affected side up. The position is maintained with a posterior sacral fixed support and an anterior fixed support against the Anterior superior iliac spine. The upper arm is rested on an arm support. The lower arm is padded especially in axillary area. A Pillow is placed between the two legs. Note the externally rotated posture of the affected side due to severe SUFE (Red arrow); B) The incision is marked on the skin. This is a straight incision centred at the anterior third of the greater trochanter and extending proximally to the level of the anterior superior iliac spine and for about $10 \mathrm{~cm}$ distally. 

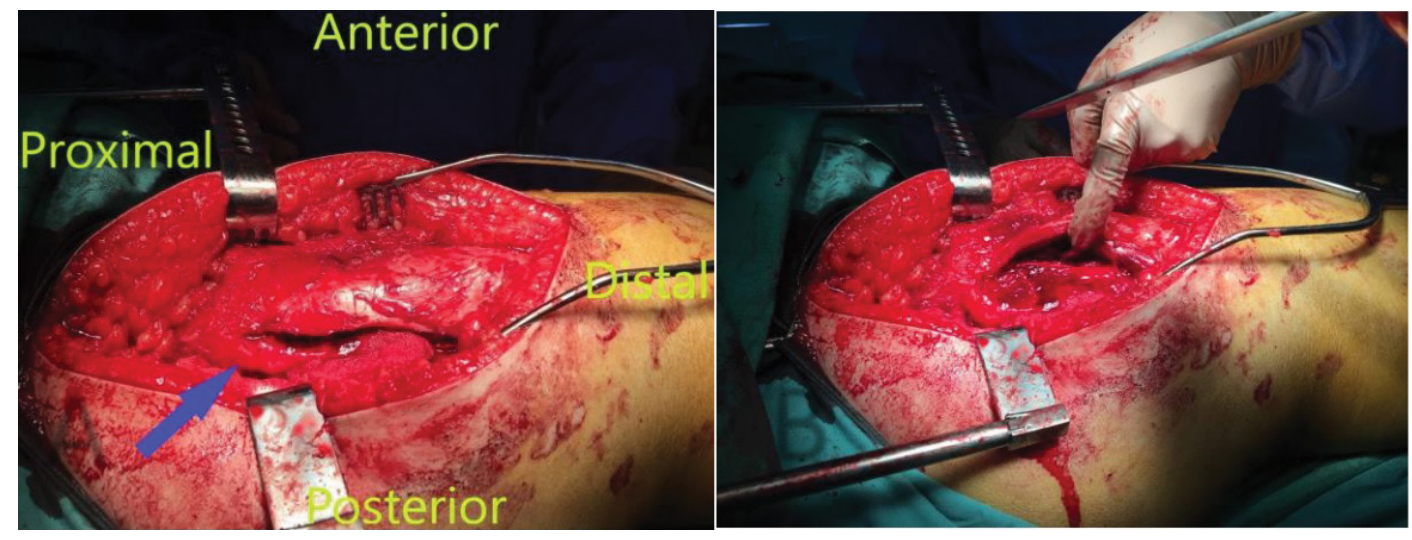

Figure 3: Trochanteric osteotomy. A) Greater Trochanter osteotomy. The view from the posterior side of the patient (The surgeon's view). A plain is developed under the vastus lateralis muscle distal to the trochanter. The osteotomy is done with oscillating saw. Note the posterior fibers of the gluteus medius muscle (Blue arrow) which are left attached. The thickness of the osteotomy should be around $1 \mathrm{~cm}$; B) The trochanteric osteotomy is mobilized with an osteotome to free the fragment and mobilized anteriorly. The thickness of the osteotomy is about $1 \mathrm{~cm}$ as shown. Note the remaining posterior fibers of gluteus medius which are left attached to the posterior aspect of the trochanter (Blue arrow).

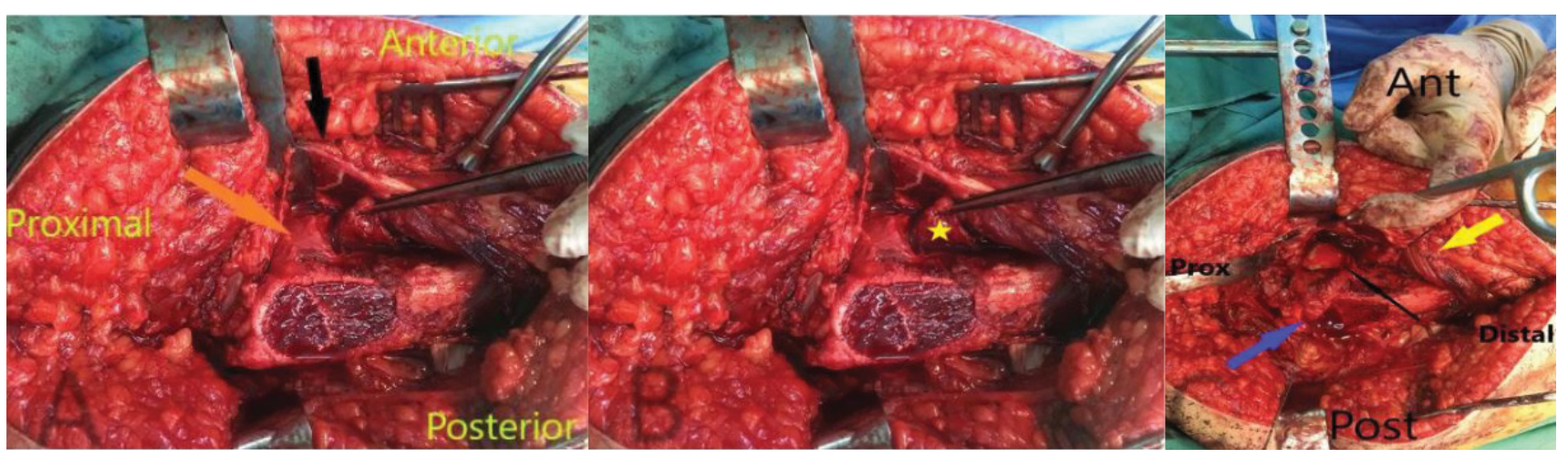

Figure 4: Capsular exposure and capsular flaps. A) Superior view. The osteotomised trochanter is displaced anteriorly (black arrow). The anterior capsule is exposed (Orange arrow). The inferior capsular flap is held between the forceps; B) Superior view- closer photo: the trochanter is displaced anteriorly. The inferior capsular flap is held between the forceps (yellow star); C) Superior view: the capsulotomy is completed in Z shape. Both capsular flaps are tagged with sutures; inferior capsular flap (yellow arrow), superior capsular flap (Blue arrow). The femoral neck is now fully exposed (Black arrow).



Figure 5: Temporary pinning of sliped femoral head before dislocation. A) The slipped femoral head is temporarily fixed to the neck with 2 thick $\mathrm{K}$ wires prior to dislocation. Failure to do this step will tear the retinacular blood supply at the time of dislocation and may cause avascular necrosis. Note the very anterior entry points of the wires on the femoral neck in order to catch the femoral head; B) Temporary pinning of the femoral head prior to dislocation. Radiological confirmation of the position of the pins in the femoral head. Also a clinical check must be done to confirm the femoral head and neck are strongly fixed and are moving as one unit before dislocation. Dislocation must be done very gently and slowly. 


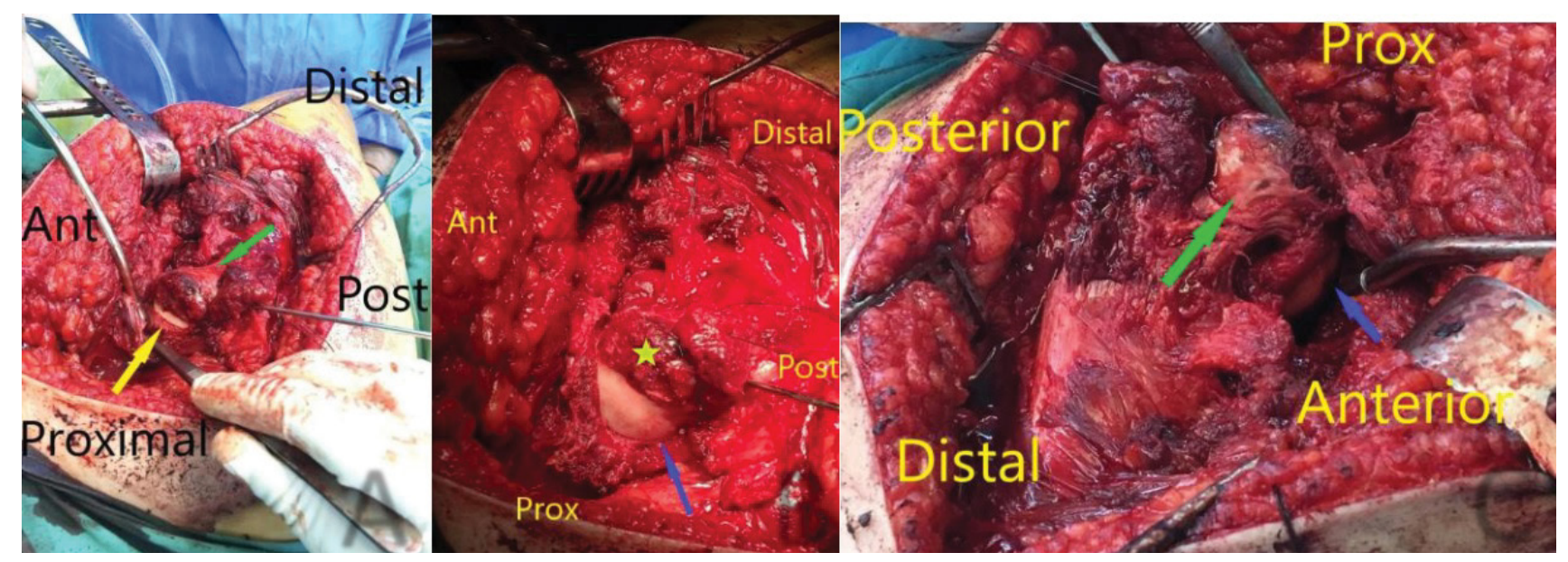

Figure 6: Severity of the slip. A) View after dislocation. Note the severity of the slip; The femoral head (yellow arrow) is severely slipped and is sitting at right angle posterior to the femoral neck (green arrow). The proximal end of the femoral neck is bare; B) The severity of the slip. The femoral head (blue arrow) is completely slipped posteriorly and the proximal part of the femoral neck is completely bare (yellow star); C) Anterior view. The femoral head (blue arrow) is severely slipped in relation to the femoral neck (Green arrow)
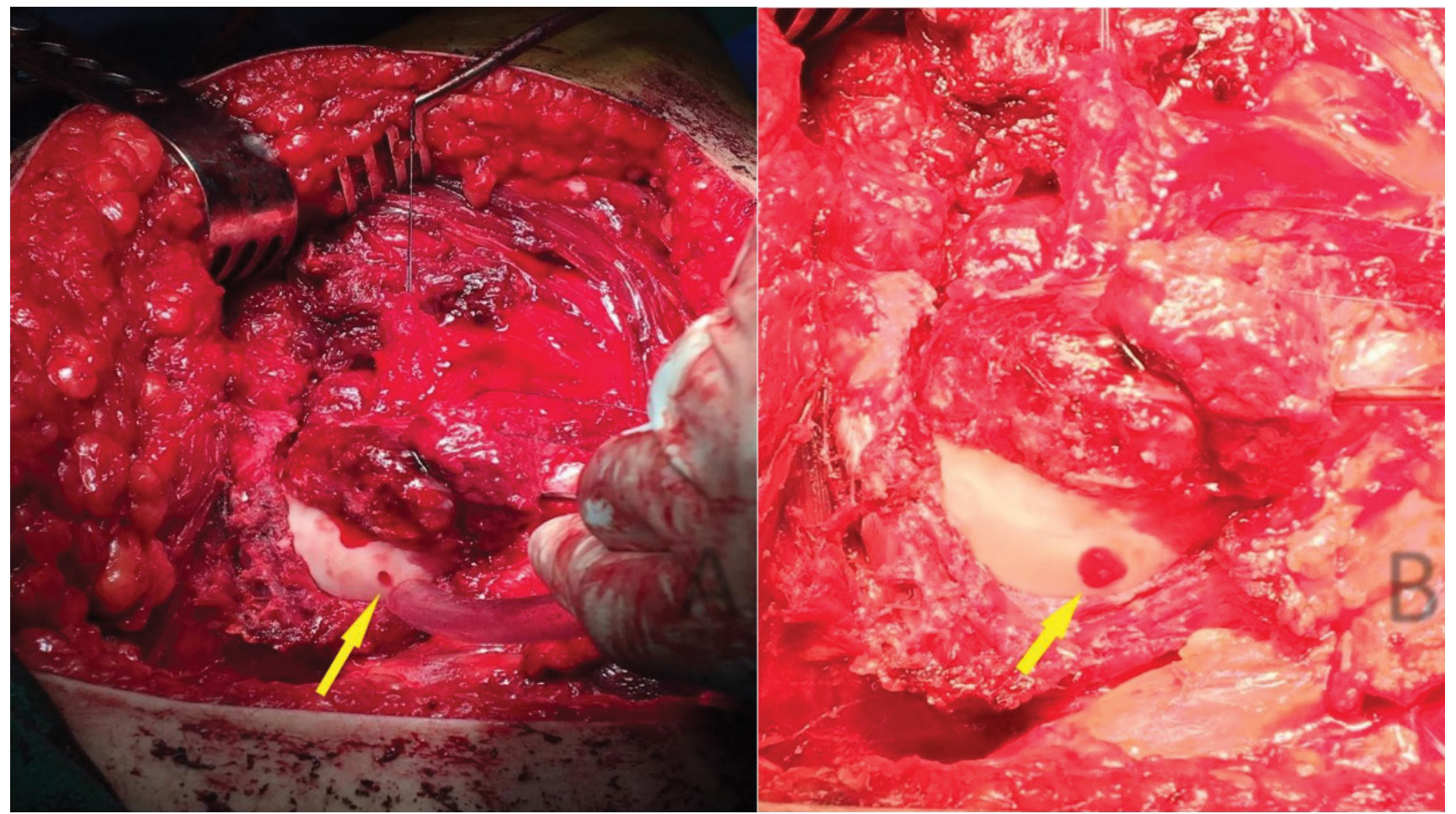

Figure 7: Checking femoral head vascularity. A) Checking the vascularity of the femoral head. A K wire is used to create a drill hole in the femoral head and suction is placed next to the hole (yellow arrow). Note that there is bleeding from the drilled hole indicating that there is some vascularity to the femoral head; B) Bleeding from the drilled hole in the femoral head indicating that the femoral head is still perfused.

\section{Materials and Methods}

We present one of our patients who presented to our department with severe SUFE and who required the modified Dunn procedure through the Ganz Safe surgical dislocation approach. We will present the initial radiological examinations and then go through the operation step by step and demonstrateeach step with intra-operative photos.

\section{Case Presentation}

An eleven year old boy with high BMI for age presented to the Emergency Department with $R$ Thigh pain with limping. He had pain for 2 months but the pain got suddenly worse over the previous 24 hours. He was unable to bear weight on the affected side. Clinical examination revealed an externally rotated posture of the Right lower limb with severe pain on any attempted movement of the hip. Initial xray was taken 


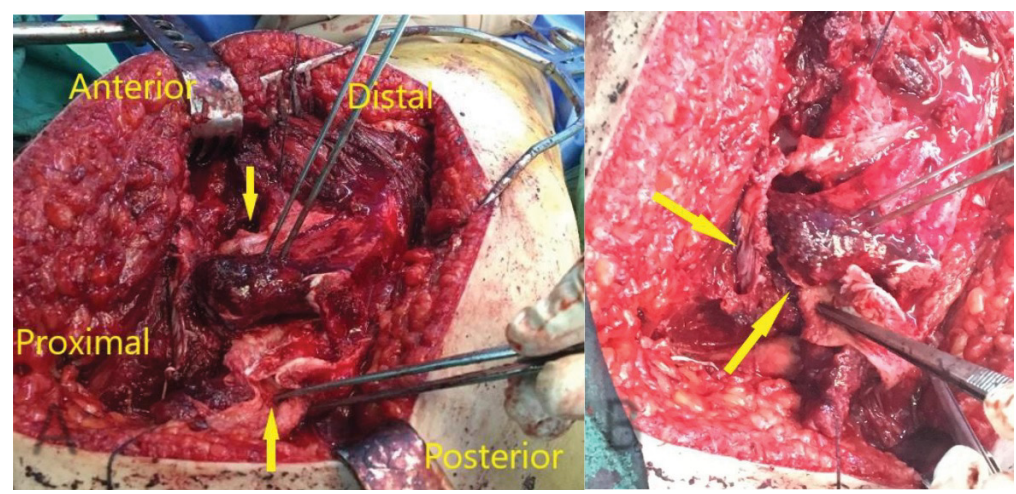

Figure 8: Retinacular flaps. A) This is a critical step in this technique and must be done very slowly and gently. The retinacular layer is split on the anterior aspect of the neck in line with anterior edge of the greater trochanter towards the proximal end of the femoral neck. The anterior flap is elevated with a periosteal elevator around the medial side. Posteriorly, the bed of the trochanter is decancelled i.e the cancellous bone removed using curettes and small gauges leaving only the thin supero-posterior conrtical rim. Then, the cortex is cracked and elevated as a single layer with the posterior retinacular flap. Special care must be taken to protect the insertion of the piriformis tendon which is near to the lateral epiphyseal artery. The flap elevation is continued until the retinacular flaps are attached only to the femoral head but not to the neck (yellow arrows); B) The retinacular flaps (yellow arrows) are completely elevated off the femoral neck all around and are attached only to the femoral head.


Figure 9: Femoral head reduction and fixation. A) After developing the flaps, the $\mathrm{K}$ wires can be removed. The remnant of the growth plate is curetted off the femoral neck and head. The next step is to prepare for gentle and easy femoral head reduction without putting any tension on the retinacular flaps and the blood supply. To do that all posterior callus must be removed. This should be done gently and slowly until all callus is removed. This is done using a combination of nibblers, curettes and rasps. After that the reduction can be done. The reduction must be gently and slowly done. If any tension is noted on the flaps then the surgeon must stop and to try to further clear the posterior neck from callus again and shorten the neck slightly if required. Note the anterior retinacular flap (yellow arrow) that is attached to the femoral head but not to the neck. The drill hole in the femoral head is still bleeding indicating the vascularity is maintained after developing the flaps. Once the femoral head is easily reducible without tension, the fixation must be prepared for before final femoral head reduction; B) The fixation must be prepared prior to final slip Reduction. 2 wires from the cannulated screw set are inserted retrograde from the central area of bare femoral neck to the lateral cortex of the proximal femur and then the femoral head is reduced and the wires advanced into the head. The position and lengths are checked using c-arm. Gentle drilling and screw advancement are done. Anterior view after slip reduction and fixation. Both retinacular flaps (yellow arrows) are intact and attached to the femoral head and are very lax without tension. The femoral head drill hole is still bleeding indicating the perfusion is maintained (Blue arrow). The screws holding the reduction are shown (Green arrow); (C): Final position after reduction and fixation. The normal relationship between the femoral head and neck is restored. Both Flaps are intact and lax (yellow arrows). Cannulated Screws used for fixationare shown (green arrow); (D): intra-operative C-arm image after fixation. The slip is anatomically reduced and two cannulated screws are holding the reduction;(E): : intra-operative c-arm image after trochanteric fixation. The femoral head is reduced. The trochanter is fixed with 2 or $33.5 \mathrm{~mm}$ fully threaded cortical screws; (F): intra-operative c-arm image showing lateral view of the hip after reduction and fixation of the slip and after fixation of the greater trochanter. 


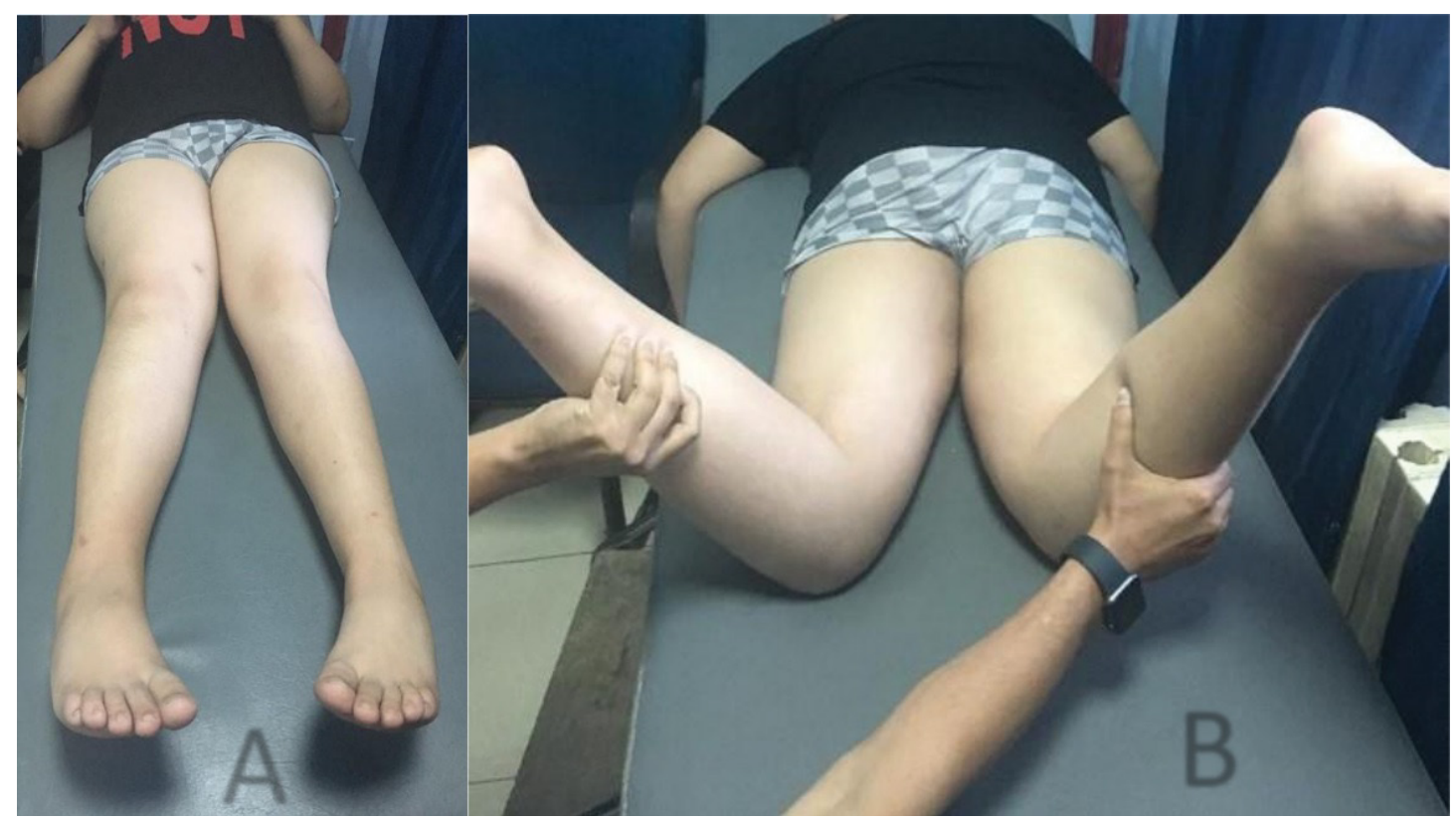

Figure 10: Outcome. A) 6-months post-operatively. The patient is pain free and mobilizing fully weight bearing. There is no leg length difference and he has a symmetrical rotational profile; B) The patient has full range of hip movement and a symmetrical rotational profile.

and was thought to be normal although it was suboptimal and as a result the patient was sent home. The following day the patient returned to the hospital with similar complains and repeat $\mathrm{x}$-rays were taken showing a severe slip. He was admitted and placed on gentle traction ( $1 \mathrm{Kg}$ ). Perfusion MRI scan was requested and showed that perfusion of the femoral head is maintained. It also showed the severity of the slip and the difficulties of pinning the slip in situ. The MRI also showed that there an element of chronicity due to the presence of some callus around the posterior femoral neck. A diagnosis of severe acute on chronic slip was made (All images are in Figure 1). The patient was operated on day 10 after admission. We present below the surgical steps demonstrated by intra-operative photos (Figure 2, Figure 3, Figure 4, Figure 5, Figure 6, Figure 7, Figure 8, Figure 9 and Figure 10).

\section{Conclusion}

Surgical Hip dislocation popularized by the Bernese group is an effective approach to deal with intra-articular hip pathology in all age groups. It is, however, a very technical approach and attention to detail is of paramount importance to avoid complications. Using this technique in treating slipped upper femoral epiphysis is even more challenging due to the nature of the disease and the already compromised femoral head blood supply. In this paper we demonstrated this technique with detailed explanation of every step as well as intraoperative photos aiming to help paediatric and young adult hip surgeons understand each step of the approach. We hope this article will encourage surgeons to use this approach instead of in situ pinning for treating severe slipped upper femoral epiphysis patients.

\section{Level of Evidence}

\section{References}

1. Lehmann CL, Arons RR, Loder RT, et al. (2006) The epidemiology of slipped capital femoral epiphysis: An Update. J Paediatr Orthop 26: 286-290.

2. Madan SS, Cooper AP, Davies AG, et al. (2013) The treatment of severe slipped capital femoral epiphysis via the Ganz surgical dislocation and anatomical reduction: A prospective study. The Bone \& Joint Journal 95: 424-429.

3. Mohammed Elmarghany, Tarek M Abd El Ghaffar, Mahmoud Seddik, et al. (2017) Surgical hip dislocation in treatment of slipped capital femoral epiphysis. Sicot J 3: 10.

4. Kai Ziebarth, Christoph Zilkens, Samantha Spencer, et al. (2009) Capital realignment for moderate and severe SCFE using a modified dunn procedure. Clin Orthop Relat Res 467: 704-716.

5. Nicola Ebert, Martin Rupprecht, Ralf Stuecker, et al. (2019) Outcome of the modified dunn procedure in severe chronic or acute on chronic slipped capital femoral epiphysis. Journal of Orthopaedic Surgery and Research 14: 349.

6. R Ganz, TJ Gill, E Gautier, et al. (2001) Surgical dislocation of the adult hip. A technique with full access to the femoral head and acetabulum without the risk of avascular necrosis. J Bone Joint Surg Br 83: 1119-1124.

7. Alessandro Masse, Alessandro Aprato, Caterina Alluto, et al. (2015) Surgical hip dislocation is a reliable approach for treatment of femoral head fractures. Clin Orthop Relat Res 473: 3744-3751.

8. M Leunig, HM Manner, L Turchetto, et al. (2017) Femoral and acetabular re-alignment in slipped capital femoral epiphysis. Journal of Children's Orthopaedics 11: 131-137. 